\title{
Unenhanced breast MRI: could it replace dynamic breast MRI in detecting and characterizing breast lesions?
}

\author{
Reham Khali|*'D, Noha Mohamed Osman, Nivine Chalabi and Enas Abdel Ghany
}

\begin{abstract}
Background: We aimed to evaluate the unenhanced MRI of the breast (UE-MRI) as an effective substitute for dynamic contrast-enhanced breast MRI (DCE-MRI) in both detecting and characterizing breast lesions. We enrolled in our retrospective study 125 females (232 breasts, as 18 patients had unilateral mastectomy) with breast mass at MRI of variable pathologies. Routine DCE-MRI protocol of the breast was conducted. We compared the conventional unenhanced images including STIR, T2, and DWIs to the DCE-MRI by two blinded radiologists, to detect and characterize breast lesions, and then we compared their results with the final reference diagnoses supplied by the histopathology or serial negative follow-ups. Sensitivity, specificity, positive predictive value (PPV), negative predictive value (NPV), and diagnostic accuracy for UE-MRI and DCE-MRI were calculated. UE-MRI results of each observer were also compared with DCE- MRI.
\end{abstract}

Results: The calculated UE-MRI sensitivity, specificity, positive predictive value, negative predictive value, and diagnostic accuracy for the first observer were $95 \%, 80 \%, 83 \%, 94 \%$, and $89 \%$ respectively, and for the second observer, they were $94 \%, 79 \%, 81 \%, 93 \%$, and $86 \%$. On the other hand, those for the DCE-MRI by the first observer were $98 \%, 82 \%, 84 \%$, $98 \%$, and $90 \%$ and were $97 \%, 81 \%, 84 \%, 97 \%$, and $89 \%$ by the second observer. The intraobserver agreement between the UE-MRI and DCE-MRI results of each observer was $94 \%$ and $95 \%$, while the interobserver agreement for each section was $97.4 \%$ for UE-MRI and $98.3 \%$ for DCE-MRI.

Conclusion: UE-MRI of the breast can be a reliable and effective substitute for breast DCE-MRI. It can be used with comparable accuracy to DCE-MRI whenever contrast administration is not feasible or contraindicated.

Keywords: Breast, UE-MRI, DCE-MRI

\section{Background}

Proper evaluation of breast lesions is crucial for early detection and management planning of breast cancer. Imaging using mammography and ultrasound (US) (despite their well-known limitations) remains the primary standard modality for assessment of breast pathologies or for screening of high-risk group [1]. Nevertheless, MRI has proven its superior sensitivity and has established its indication in the daily practice scenarios such as mammographically occult lesions in dense breasts, discrepancy in size (mammography/ultrasound/clinical), suspected multifocal disease, lobular carcinomas, prior

\footnotetext{
* Correspondence: Rohamawad@hotmail.com

Radiodiagnosis Department, Faculty of Medicine, Ain Shams University, Abbaseya Square, Cairo 11566, Egypt
}

primary chemotherapy treatment, assessment of breast implant integrity, and follow-up of response of treatment $[2,3]$. Lesion categorization with The Breast Imaging Reporting and Data System (BI-RADS) is a widely accepted method and is applied for use with mammography, breast US, and MRI as well. It aims to pick up the morphologically suspicious lesions to justify the referral for biopsy; in spite of this, around $70-80 \%$ of breast biopsies reveal benign conditions. This is mainly attributed to biopsies of low risk breast lesions, namely BI-RADS 3 and 4A [4]. Although the former one often indicates the need for a 6-month follow-up visit, anxiety related to uncertainty usually leads to biopsy. It is well known how the biopsy procedure afflict the patients psychologically and even sometimes financially, and in order to lessen these unnecessary costs, better differentiation between 
benign and malignant pathologies is required $[5,6]$. Breast imaging using the dynamic contrast-enhanced MRI (DCE-MRI) had been introduced and has become an important tool in the workup of breast lesions. It is based on studying the enhancement kinetics of a lesion after intravenous injection of contrast agent. The washout curve pattern is believed to be a good predictive for malignancy in which neo-angiogenesis and increased tissue permeability is the underlying mechanism of such a pattern [3]. This technique has subsequently increased MRI sensitivity in cancer detection [7, 8]. However, injection of contrast is contraindicated in some cases like renal impairment, and even if it is injected, this has its drawbacks like allergic reaction, contrast-induced systemic fibrosis, time, and financial cost $[9,10]$. For this, new attention is paid to unenhanced MRI (UE-MRI) of the breast using the diffusion-weighted (DW), short tau inversion recovery (STIR), and T2-weighted sequences as an alternative to DCE-MRI.

\section{The aim of this work}

It was to evaluate the unenhanced MRI of the breast (UE-MRI) as an effective substitute for dynamic contrast-enhanced breast MRI (DCE-MRI) in both detecting and characterizing breast lesions.

\section{Methods}

\section{Patients}

We retrospectively enrolled 125 females with breast mass lesions of variable pathologies detected at MRI breast exams, during the period from January 2016 to December 2016. Eighteen patients had underwent unilateral mastectomy. All the included scans were of a mass lesion that was either histopathologically proven or had a negative follow-up MRI scan that had been 2 years later done at our center. Patients who had non-mass lesions were excluded from this study. Patients who had recently-prior to MRI scan-underwent biopsy or on chemotherapy were also excluded due to possible alteration of lesional morphology. The local Ethics Committee had approved this retrospective study, and patients' consents were waived due to its retrospective nature.

\section{Breast MRI technique}

All MRI examinations were performed using 1.5 Tesla Achieva (Philips Medical Systems, Edinburgh, the Netherlands). All patients were examined in the prone position using breast array coil. Our institutional routine breast MRI protocol was conducted without modification. A survey sequence was followed by axial T1 and T2WI fast spin-echo (FSE) sequence, axial and sagittal STIR fat suppressed images, and single-shot echo-planar DW imaging for both breasts prior to contrast administration in order to avoid signal alteration by the injected gadolinium. Tri-directional diffusion gradients were used with $b$ values 0,400 , and $800 \mathrm{~s} / \mathrm{mm}^{2}$ to increase sensitivity to cellular packing; respiratory triggering was used for better resolution. Number of excitations was 2, matrix $256 \times 256$, field of view $34 \mathrm{~cm}$, slice thickness 3 $\mathrm{mm}$, and gap 0 . Lastly, dynamic contrast-enhanced (DCE) sequence was performed in order to avoid possible alteration of the T2 SI or apparent diffusion coefficient (ADC) values. Axial and sagittal post-contrast T1WI fat-suppressed images were obtained after a bolus of gadopentetate dimeglumine was injected (at a dose of $0.1 \mathrm{mmol}$ per kilogram of body weight and a rate of 2 $\mathrm{mL} / \mathrm{s}$ ), followed by $20 \mathrm{~mL}$ saline flush. One acquisition was performed prior to contrast administration, and three acquisitions were performed over a period of 6 min after intravenous contrast material injection. At Easy Vision, Philips Medical Systems workstation, ADC values were measured. The ADC values for the different $b$ values $\left(0,400\right.$, and $\left.800 \mathrm{~s} / \mathrm{mm}^{2}\right)$ were obtained by placing four regions of interest (ROIs) on the ADC map in the area with pathological enhancement. The (ROI) area was between 1 and $2 \mathrm{~cm}^{2}$, and the final ADC value was the average of the four measured values for each $b$ value. Time intensity curves were generated by drawing region of interest (ROI) at the area of maximum higher visual enhancement, and meticulous care was taken to include the solid portion of the lesion and to avoid any central cystic regions. The kinetic curves of the DCE sequence were generated and were categorized into three pattern types (persistent, plateau, and washout pattern) [3].

\section{MRI exams interpretation}

Each breast MRI exam was divided into two sections: UE-MRI images (including STIR, T2, and DWIs) and DCE-MRI images. Two consultant radiologists in women (breast) imaging blindly interpreted both sections separately with 1 -month interval. Each time, they were asked to detect and characterize breast lesions each time. They had assessed the UE-MRI scans and had to describe how much easily the lesion was visible, to categorize the lesion in terms of BI-RADS classification according to its morphology using the T2WI and STIR images (with special emphasis on lesion margin as well its signal intensity in relation to the surrounding glandular tissues), and to inspect DWI for hyperintense lesions and-as reviewed in the literature [11]-lesions with ADC values less than $1.3 \times 10^{-3} \mathrm{~mm}^{2} / \mathrm{s}$ which were considered suspicious for malignancy. They also had to study the DCE-MRI scans and to evaluate the dynamic enhancement features of the mass lesion. Afterwards, we compared their results with the final reference diagnoses supplied by the histopathology of breast lesions or serial negative follow-ups accordingly. 


\section{Statistical analysis}

Statistical analysis was performed by using software (SPSS, version 20.0; SPSS, Chicago). Sensitivity, specificity, positive predictive value (PPV), negative predictive value (NPV), and diagnostic accuracy for UE-MRI and DCE-MRI were calculated. Intra- and interobserver agreement was assessed. UE-MRI results were also compared with DCE- MRI.

\section{Results}

Our study included 125 females, 18 of them had unilateral mastectomy (232 breast scans). Their age ranged from 23 to 68 years (average 42 years). According to the reference standard (lesion histopathology or negative serial 2 years follow-ups), 115 lesions were proved to be malignant and 68 were benign, while 49 breasts had no lesions.

Studying the MRI images, no abnormalities were depicted in either the UE-MRI or DCE-MRI in the 49 scans. These were confirmed to be normal in their follow-up examination. On the other hand, 183 of the encountered scans revealed breast lesions which were subjected to further categorization radiologically into either malignant or benign. Table 1 sums up the results.

Comparing the lesion visibility in DWI and DCE- MRI, the subtly visible lesions were $5 \%$ in the UE scan and $2 \%$ in DCE scans. Table 2 shows the distribution of these lesions. Studying lesion border in the T2WI revealed 78\% of malignant lesions (90 out of 115) had irregular margins. This was also noted in $25 \%$ of benign lesions (17 out of 68 ) (Table 3 ). In T2WI, the most malignant lesions (96\%) (110 out of 115 ) displayed hypointense signal intensity; oppositely, most of benign lesions (94\%) (64 out of 68) were hyperintense (Table 3). Regarding ADC values, benign lesions had significantly higher values than the malignant ones. The average ADC values were $1.59 \pm 0.35 \times 10^{-3}(\mathrm{SD}) \mathrm{mm}^{2} / \mathrm{s}$ in benign lesions and $1.01 \pm 0.31(\mathrm{SD}) \times 10^{-3} \mathrm{~mm}^{2} / \mathrm{s}$ in malignant ones.

After reviewing the UE-MRI, the observers reported that 132 lesions (72\%) by the first observer and 133 (73\%) by the second one (out of 183) were suspected for malignancy due to ill-defined outlines, high SI in T2, STIR, and DW images, and low ADC value. However, comparing these with the reference diagnoses, 23 (13\%) and $25(14 \%)$ lesions of them respectively were confirmed to be benign rather than malignant. Hence, 109 (60\%) and 108 (59\%) were the true positive for the observers respectively, while 23 and 25 were their falsepositive results. Moreover, 51 lesions $(28 \%)$ by the first observer and 50 lesions (27\%) by the second observer (out of the 183) were reported as benign ones, yet $6(3 \%)$ and 7 (4\%) of those respectively were proved histopathologically to be malignant and so counted as the false negatives of the UE-MRI. The calculated UE-MRI sensitivity, specificity, positive predictive value, negative predictive value, and diagnostic accuracy for the first observer were as follows respectively: 95\%, 80\%, 83\%, $94 \%$, and $89 \%$. Similarly, for the second observer, they were $94 \%, 79 \%, 81 \%, 93 \%$, and $86 \%$ respectively.

Later on, by reviewing the DCE-MRI, lesions showed mass enhancement, and their kinetics was studied. Each consultant separately decided that 134 lesions (out of 183) were considered as malignant lesions, yet, 21 and 22 lesions of those were established to be of benign nature (i.e., 113 and 112 were the true positive of the observers, while 21 and 22 were their false positives). Furthermore, 49 lesions (out of the 183) were reported by each radiologist as benign lesions, yet 2 and 3 of those were proved by biopsy to be malignant and so counted as the false negatives of the DCE-MRI for each observer. The calculated DCE-MRI sensitivity, specificity, positive predictive value (PPV), negative predictive

Table 1 The distribution of the results after reviewing the scans by two observers

\begin{tabular}{|c|c|c|c|c|c|c|}
\hline \multicolumn{5}{|c|}{232 Breast MRI scans } & & \\
\hline \multirow{11}{*}{$\begin{array}{l}49 \text { No } \\
\text { Lesion } \\
\text { neither } \\
\text { in UE nor } \\
\text { DCE MRI }\end{array}$} & \multicolumn{4}{|c|}{$\begin{array}{c}183 \text { with lesions } \\
\text { (Histo-pathologically: } 115 \text { malignant }+68 \text { benign) }\end{array}$} & & \\
\hline & \multicolumn{2}{|c|}{ UE-MRI } & \multicolumn{2}{|c|}{ DCE-MRI } & & \\
\hline & \multirow[t]{4}{*}{ Observer 1} & True +ve $=109$ & \multirow[t]{4}{*}{ Observer 1} & True +ve $=113$ & \multirow{4}{*}{$\begin{array}{l}6 \\
\text { L̊ } \\
0\end{array}$} & \multirow{8}{*}{ 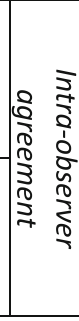 } \\
\hline & & False +ve $=23$ & & False $+v e=21$ & & \\
\hline & & True -ve $=45$ & & True -ve $=47$ & & \\
\hline & & False - ve $=6$ & & False - ve $=2$ & & \\
\hline & \multirow[t]{4}{*}{ Observer 2} & True $+v e=108$ & \multirow[t]{4}{*}{ Observer 2} & True $+v e=112$ & \multirow{4}{*}{$\frac{9}{80}$} & \\
\hline & & False $+v e=25$ & & False $+v e=22$ & & \\
\hline & & True - ve $=43$ & & True - ve $=46$ & & \\
\hline & & False - ve $=7$ & & False - ve $=3$ & & \\
\hline & $\begin{array}{l}\text { Inter-observer } \\
\text { agreement: }\end{array}$ & $97.4 \%$ & $\begin{array}{l}\text { Inter-observer } \\
\text { agreement: }\end{array}$ & $98.3 \%$ & & \\
\hline
\end{tabular}


Table 2 Comparing lesion visibility in DWI and CE-MRI sequences

\begin{tabular}{lllll}
\hline & & Subtly visible & Clearly visible & Total \\
\hline DWI & Benign lesions & 8 & 60 & 68 \\
& Malignant lesions & 2 & 113 & 115 \\
\multirow{2}{*}{ CE-MRI } & Benign lesions & 3 & 65 & 68 \\
& Malignant lesions & 1 & 114 & 115 \\
\hline
\end{tabular}

value (NPV), and diagnostic accuracy for the first observer were as follows respectively: $98 \%, 82 \%, 84 \%, 98 \%$, and $90 \%$. On the other hand, for the second observer, they were $97 \%, 81 \%, 84 \%, 97 \%$, and $89 \%$.

Intraobserver agreement between UE-MRI and DCEMRI results of each observer was $94 \%$ and $95 \%$, while interobserver agreement for each section was $97.4 \%$ for UE-MRI and $98.3 \%$ for DCE-MRI. Figures 1, 2, 3, 4 and 5 represent some encountered cases.

\section{Discussion}

MRI is a well-established modality in breast imaging; moreover, at present, the DCE-MRI is considered the gold standard technique in patients with breast lesions, with a reported sensitivity of $95-99 \%$ in detecting invasive cancer and of $80 \%$ in detecting ductal carcinoma in situ. It allows lesions assessment morphologically as well as semi-quantitatively by the enhancement kinetics [12-17]. So, absence of the peculiar features of malignancy in the enhanced sequence may alleviate the need of unnecessary breast biopsy [18]. However, the DCE-MRI can be time consuming, costly, or unavailable, plus in some inevitable circumstances, contrast administration can be a concern, undesired, or even contraindicated $[10,19]$. Hence, with the evolution of new sequences like DWI, initiatives in setting up new protocols, alternative to DCE-MRI, has emerged. One of these protocols is the integration of DWI with T2WI and STIR images; this way, morphological information provided by the high spatial resolution of the T2WI is incorporated with the functional data offered by DWI.

Our study aimed to evaluate the role of UE-MRI of the breast in detecting and characterizing breast lesions compared to DCE-MRI of the same breast lesions. We found

Table 3 Lesion signal intensity and margin definition as noted in T2Wls

\begin{tabular}{llll}
\hline & & Benign lesions & $\begin{array}{l}\text { Malignant } \\
\text { lesions }\end{array}$ \\
\hline Lesions margins & Well defined & 51 & 25 \\
& III defined & 17 & 90 \\
Signal intensity & Hypointense & 4 & 110 \\
& Hyperintense & 64 & 5 \\
\hline
\end{tabular}

out that UE-MRI has close results to DCE-MRI with strong interobserver agreement in lesion characterization.

Although all lesions were depicted in both of DCEMRI and UE-MRI in our study, we noted that the former revealed the lesions slightly more readily and confidently than the UE-MRI. Additionally, we found that the subtly visible lesions in DWI were more of benign nature than being malignant, and this could be assigned to the fact that most malignant pathologies caused diffusion restriction and so were relatively easily spotted than the isointense to hypointense benign ones. Other reasons for this could be field in-homogeneity or small and non-mass lesions. However, careful inspection for associated abnormalities like skin changes and lymphadenopathies, plus reviewing the other UE-MRI images (STIR sequence in particular) side by side the DWI, assisted in raising the overall confidence about lesion localization and overcame the possibility of the lesion being overlooked in DWI. Some studies had also reported that DWI had a relatively reduced diagnostic performance in lesions measuring $1 \mathrm{~cm}$ or less leading to more false-negative results [20].

For lesion characterization using the ADC values, although we found some overlapping between benign and malignant lesions, the mean average of the benign ones was significantly higher than malignant ones, and thus adding this parameter was profitable in increasing the diagnostic accuracy of UE-MRI in our study. Different studies had set different cutoff ADC values depending on their scanning parameters; nevertheless, a cutoff value of $1.30 \times 10^{-3} \mathrm{~mm}^{2} / \mathrm{s}$ for ADC had yielded $89.1 \%$ sensitivity and $100 \%$ specificity for the differentiation between benign and malignant lesions [8]. Some studies had questioned the value of DWI and ADC and discovered that it is a beneficial tool in lesion characterization and even in treatment monitoring and planning. ADC values showed strong correlation with lesion histology being notably lower in malignant lesions; also, the highest values were recorded in human epidermal growth factor receptor (HER2)-enriched tumors, while low ADC had suggested progesterone receptor (PR) negativity [11, 21]. Additionally, we found that lesion signal intensity in T2WI was a helpful indicative of its nature, as most of malignant lesions were hypointense in T2WI, opposing most benign lesions which displayed high signal intensity. However, if the malignant lesion had cystic necrosis, hemorrhagic changes, fatty component, mucinous, myxoid, or edematous stroma, it could display high T2 signal intensity mimicking benign pathologies [22]. Moreover, if a benign lesion was abundant with fibrosis or inflammatory changes, e.g., fibrocystic changes, epithelial hyperplasia, granulomatous inflammations, papilloma, fibroadenoma, and sclerosing adenosis, it might exhibit T2 hypointensity and low ADC values simulating 


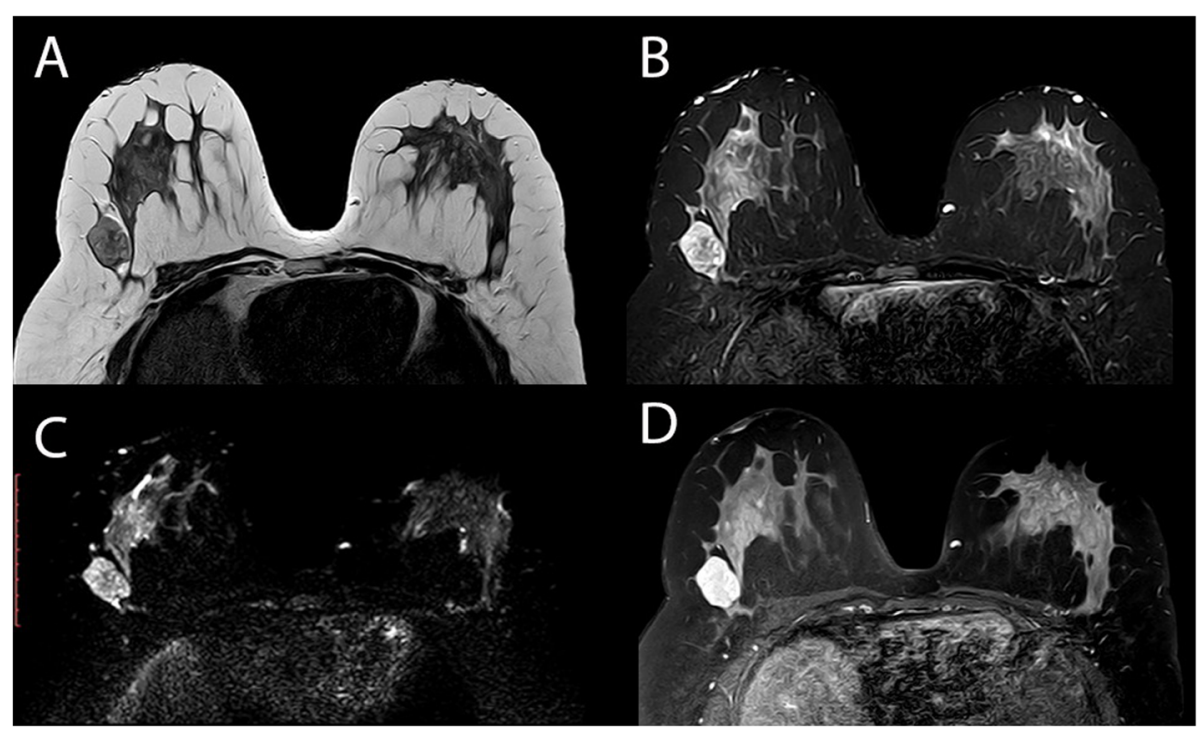

Fig. 1 A case of pathologically proven fibroadenoma in the right breast. It had a well-defined regular margin and of low T1 SI, high SI in T2WI (a) and STIR (b), and restricted diffusion in DWI (c). ADC value was $1.39 \times 10^{-3} \mathrm{~mm}^{2} / \mathrm{s}$. It exhibited homogenous contrast enhancement in DCE-MRI (d)

radiologically the malignant lesion [23]. Special attention should also be paid to the suppressed lesions in STIR sequence, knowing this sequence is not fat selective and the signal suppression counts on T1 relaxation of tissue. Subsequently, differentiating hemorrhagic versus fatty lesions on STIR images solely could be problematic [24]. Our study also confirmed the established malignancy features namely the ill-defined borders. Yet, counting solely on this manifestation could be misleading, as there was overlapping between benign and malignant lesions, i.e., $25 \%$ of benign lesions had illdefined margins while $22 \%$ of pathologically proven malignant lesions had well-defined margin radiologically (Figs. 4 and 5). It had also been concluded by

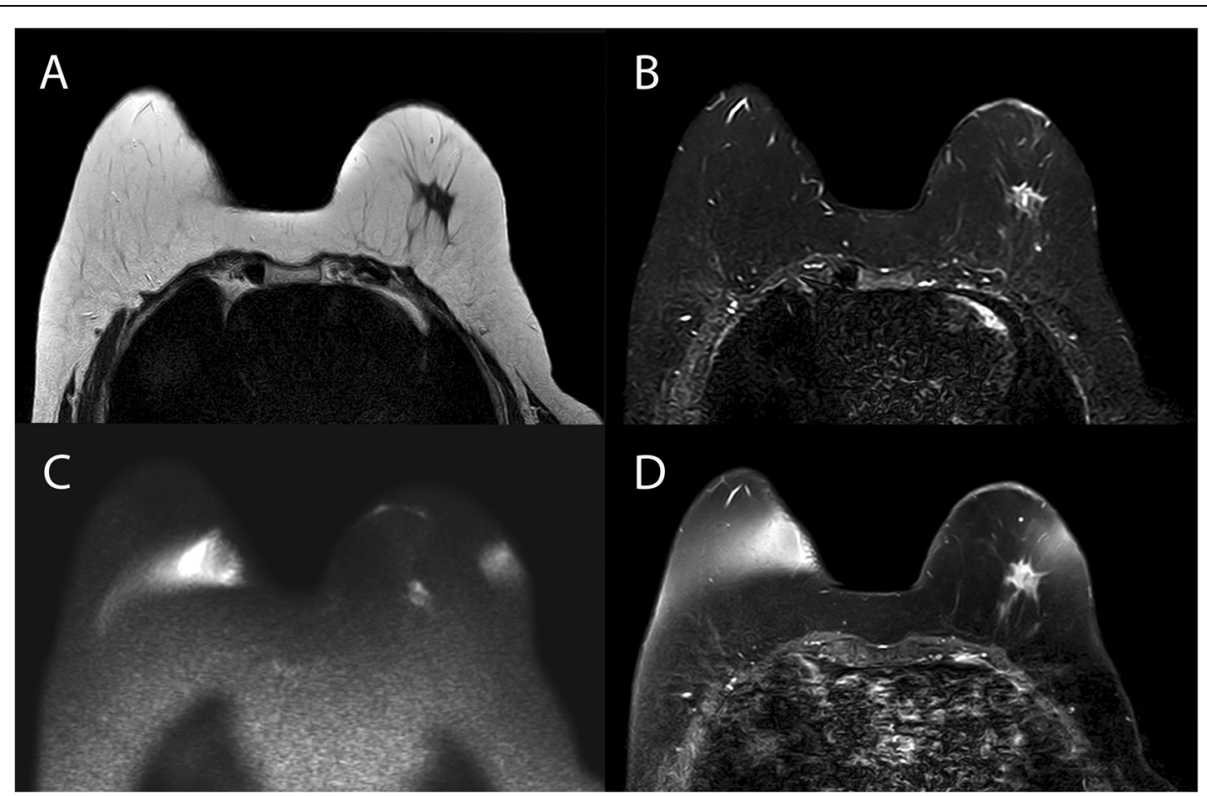

Fig. 2 A case of pathologically proven invasive duct carcinoma of the left breast. It displayed the classic appearance of an irregular speculated mass with low T1 and T2 SI (a), high SI in STIR (b), restricted diffusion in DWI (c), and heterogenous enhancement in post-contrast scans (d). It had type 3 kinetic curve consistent with malignancy. Areolar skin edema and thickening was also noted 


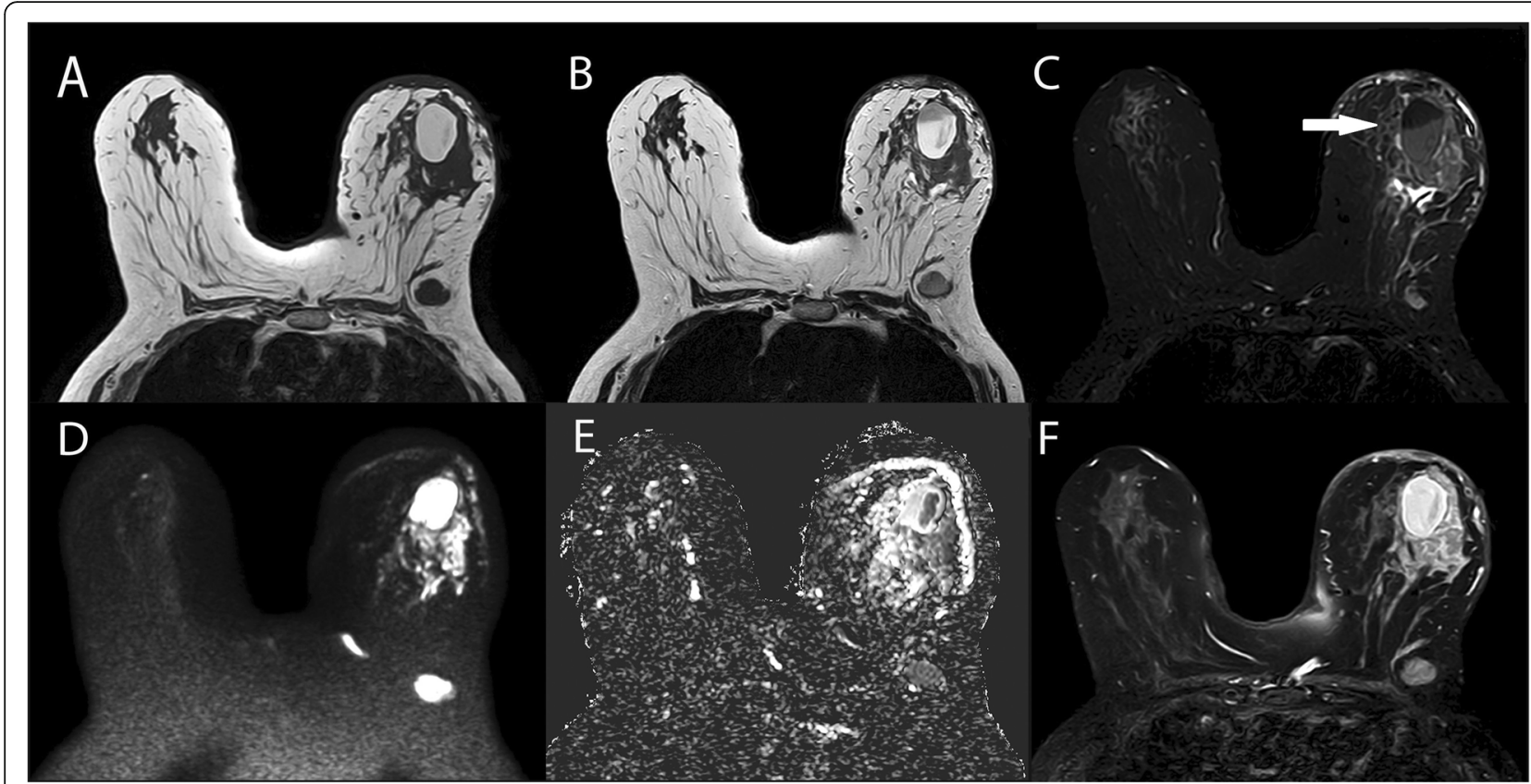

Fig. 3 In the left breast, there was an irregular mass of iso T1 SI (a), intermediate high T2 SI (b), high STIR (c) with strong restricted diffusion in DWI (d), low ADC value (e), and strong enhancement in post-contrast scan (f). It was associated with skin thickening and edema as well as suspicious axillary lymph node. Eccentric within the mass was a thin walled cyst of high T1 SI (a), high T2 SI (b), and bright in DWI (d), but for being suppressed in STIR sequence (c, arrowed), the probability of fatty versus hemorrhagic content was equivocal. In the post-contrast sequence (f), in which fat frequency-selective saturation technique was used, the cyst was not suppressed indicating it was hemorrhagic and not fatty. Also, an intracystic extension of the malignant lesion could not be defined within the hyperintense contents. The histopathology confirmed it was invasive duct carcinoma, engulfing a complicated cyst

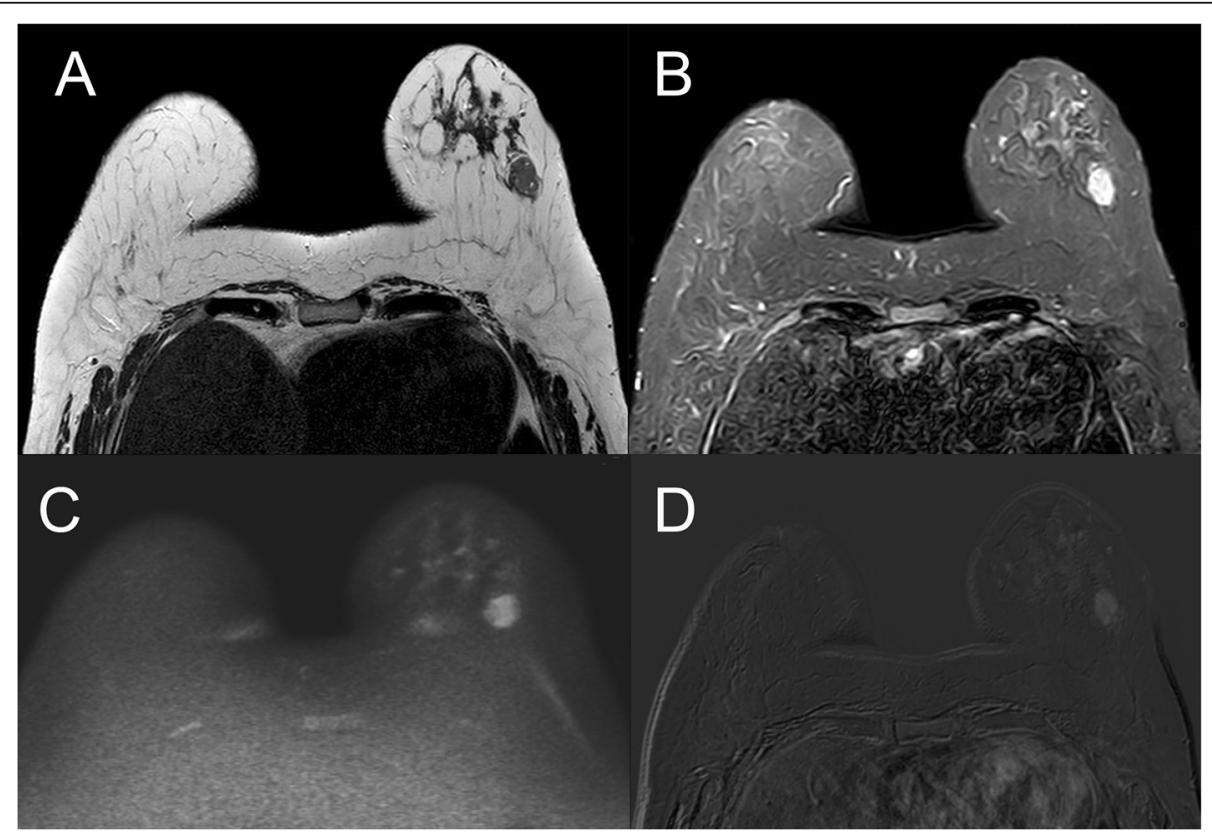

Fig. 4 A well-defined mass lesion was noted in left breast. It was of intermediate SI in T2WI (a), high in STIR (b), mildly restricted in DWI, with homogenous enhancement (e), and plateau-type kinetic curve in DCE-MRI. Yet, histopathology revealed it was invasive duct carcinoma 


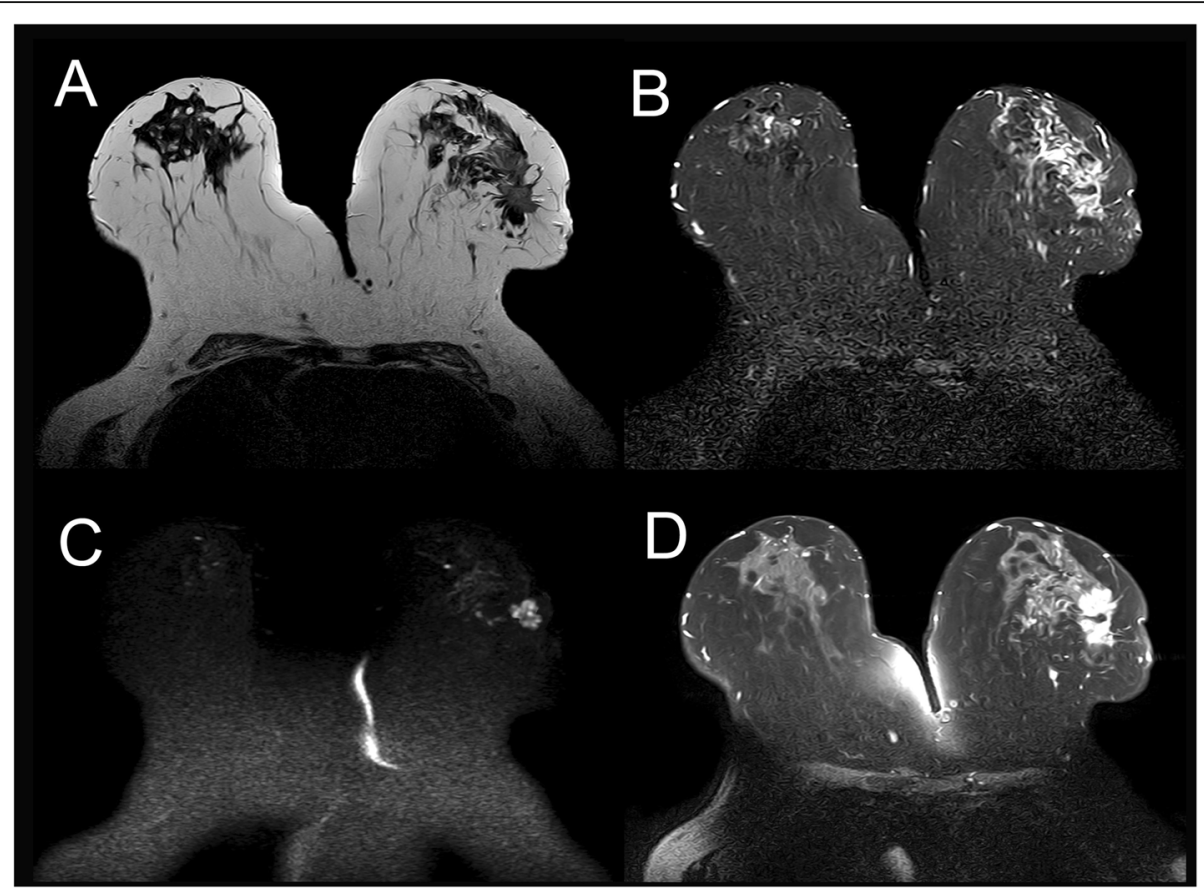

Fig. 5 In the left breast, a lobulated mass lesion of spiculated margin was noted. It exhibited intermediated SI in T2WI (a), high in STIR (b), and restricted in DWI (c). The ADC value was $0.7 \times 10^{-3} \mathrm{~mm}^{2} / \mathrm{s}$. Intense enhancement was noted in post-contrast scan (d) and the kinetics curve was of type 3. This was agreeing with biopsy result which asserted it was lobular carcinoma

others that this sign was statistically significant but still unreliable in differentiating between benign and malignant pathologies [25].

Our results were concordant with multiple studies. Belli et al. [20] had evaluated the DWI and STIR in comparison to lesion histopathology by two observers. For rater 1, STIR and DWI specificity was $99.3 \%$ and $95.7 \%$; for rater 2, it was $99.3 \%$ and $96.4 \%$. STIR and DWI sensitivity was $76.5 \%$ and $76.5 \%$ for rater 1 , and $77.5 \%$ and $77.6 \%$ for rater 2 . They had reported that UE-MRI had overall good diagnostic performance especially with lesions $\geq 1 \mathrm{~cm}$ in size, regardless its location with very good interobserver agreement for STIR and DWI. They concluded that UE-MRI can be employed where contrast injection is contraindicated. Another similar study was done by Telegrafo et al. [26]. They compared the UEMRI-including the T2, STIR WI, and DWI with background body signal suppression (DWIBS)-with the DCE-MRI and the histological findings. They reported that breast UE-MRI is an effective alternative tool to CE-MRI to evaluate breast lesions with no statistically significant difference between them. They also mentioned that STIR and DWIBS were beneficial in lesion detection while T2WI and ADC values in lesion characterization. Their calculated sensitivity, specificity, diagnostic accuracy, and PPV and NPV values for the UE-MRI was $94 \%, 79 \%, 86 \%, 79 \%$, and $94 \%$, respectively; for DCE-MRI, it was $98 \%, 83 \%, 90 \%, 84 \%$, and $98 \%$, respectively. In another research, Kul et al. [27] found that UE-MRI (combining T2 and DWI)-instead of immediate biopsy-may lower the negative biopsy rates in low-risk breast masses (namely B-RADS 3 and 4A). They reviewed UE-MRI images and categorized the lesions into benign and malignant then compared that into the histology results. They found out that UE-MRI had 91\% specificity and 99\% NPV for detection of breast cancer. A further research was carried out by Baltzer et al. [25]. Two observers assessed the UE-MRI diagnostic performance in comparison to DCE-MRI. It was revealed that there was no significant difference between the two methods and observers. They also found that lesion visibility was less in UE-MRI resulting in more false results, but lesion size measurement was very close in all sequences. Trimboli et al. [28] also evaluated the combined STIR, T2WI, and DWI sequences in comparison to pathology and negative follow-up by two blinded radiologists. They deduced that UE-MRI protocol had sensitivity and specificity of $76-78 \%$ and $90 \%$ in cancer detection respectively with nearly matched interreader agreement. Furthermore, Moschetta et al. [29] reported that T2WI and STIR sequences can be a valid tool for spotting occult inflammatory breast cancer. In comparison to biopsies results, these sequences' calculated sensitivity, specificity, diagnostic accuracy, and PPV and NPV values was $86 \%, 100 \%, 96 \%, 100 \%$, and $94 \%$. 
The limitation of our study is that the malignant encountered lesions were nearly double the benign ones. This could be attributed to our inclusive criteria, by which we were adherent; the included lesions must have their histopathology results available. Since evidently benign lesions would not be biopsied, subsequently fewer benign ones had been included.

\section{Conclusion}

UE-MRI of the breast can be a valid, reliable, and effective substitute for breast DCE-MRI. It can be used with comparable accuracy to DCE-MRI whenever contrast administration is not feasible or contraindicated.

\section{Abbreviations}

ADC: Apparent diffusion coefficient; BI-RADS: The Breast Imaging Reporting and Data System; DCE-MRI: Dynamic contrast-enhanced magnetic resonance imaging; DWl: Diffusion weighted imaging; NPV: Negative predictive value; PPV: Positive predictive value; STIR: Short tau inversion recovery; UEMRI: Unenhanced magnetic resonance imaging

\section{Acknowledgements}

Not applicable, as all participants are co-authors for this research.

\section{Authors' contributions}

$\mathrm{NMO}$ designed the work and helped in the data collection and writing of the manuscript. NC and EAG worked on the data collection, scan reviewing, and interpretation. RK contributed in the data analysis and writing of the manuscript. All authors have read and approved the final manuscript.

\section{Funding}

All authors had no fund for this research.

\section{Availability of data and materials}

All is available with the authors upon request.

\section{Ethics approval and consent to participate}

The protocol was reviewed and approved by the Local Ethics Committee of Ain Shams University. It ruled that no formal ethics approval was required in this retrospective study, and so no reference number was given by the IRB.

\section{Consent for publication}

Although the manuscript of our retrograde study contains no individual information and the images are unidentifiable, yet, a written consent for publication was obtained for these cases.

\section{Competing interests}

The authors declare that they have no competing interests.

Received: 11 October 2019 Accepted: 3 December 2019

Published online: 10 January 2020

\section{References}

1. Menezes GLG, Knuttel FM, Stehouwer BL, Pijnappel RM, Bosch MAAJ (2014) Magnetic resonance imaging in breast cancer: a literature review and future perspectives. World J Clin Oncol 5(2):61-70

2. Leithner D, Wengert GJ, Helbich TH, Thakur S, Ochoa-Albiztegui RE, Morris EA et al (2018) Clinical role of breast MRI now and going forward. Clin Radiol 73:700-714

3. Helbich TH (2000) Contrast-enhanced magnetic resonance imaging of the breast. Eur J Radiol 34(3):208-219

4. Wiratkapun C, Bunyapaiboonsri W, Wibulpolprasert B, Lertsithichai P (2010) Biopsy rate and positive predictive value for breast cancer in BI-RADS category 4 breast lesions. J Med Assoc Thail 93:830-837

5. Liang W, Lawrence WF, Burnett CB, Hwang YT, Freedman M, Trock B et al (2003) Acceptability of diagnostic tests for breast cancer. Breast Cancer Res Treat 79:199-206
6. Crowe JP, Rim A, Patrick R, Rybicki L, Grundfest S, Kim J et al (2002) A prospective review of the decline of excisional breast biopsy. Am J Surg 184:353-355

7. Morrow M, Waters J, Morris E (2011) MRI for breast cancer screening, diagnosis, and treatment. Lancet 378(9805):1804-1811

8. Elsamaloty H, Elzawawi MS, Mohammad S, Herial N (2009) Increasing accuracy of detection of breast cancer with 3-T MRI. AJR 192(4):1142-1148

9. Agarwal R, Brunelli SM, Williams K, Mitchell D, Feldman HI, Umscheid CA (2009) Gadolinium-based contrast agents and nephrogenic systemic fibrosis: a systematic review and metaanalysis. Nephrol Dial Transplant 24:856-863

10. Bellin MF, Van Der Molen AJ (2008) Extracellular gadolinium-based contrast media: an overview. Eur J Radiol 66:160-167

11. Bozkurt TB, Koç G, Sezgin G, Altay C, Gelal MF, Oyar O (2016) Value of apparent diffusion coefficient values in differentiating malignant and benign breast lesions. Balkan Med J 33:294-300

12. Huang W, Fisher PR, Dulaimy K, Tudorica LA, O'Hea B, Button TM (2004) Detection of breast malignancy: diagnostic MR protocol for improved specificity. Radiology 232:585-591

13. Kuhl CK (2000) MRI of breast tumors. Eur Radiol 10:46-58

14. Baum F, Fischer U, Vosshenrich R, Grabbe E (2002) Classification of hypervascularized lesions in CE MR imaging of the breast. Eur Radiol 12:1087-1092

15. Hoffmann U, Brix G, Knopp MV, Hess T, Lorenz WJ (1995) Pharmacokinetic mapping of the breast: a new method for dynamic MR mammography. Magn Reson Med 33:506-514

16. Kuhl CK, Mielcareck P, Klaschik S, Leutner C, Wardelmann E, Gieseke J et al (1999) Dynamic breast MR imaging: are signal intensity time course data useful for differential diagnosis of enhancing lesions? Radiology 211:101-110

17. Orel SG (1998) High-resolution MR, imaging for the detection, diagnosis, and staging of breast cancer. RadioGraphics 18:903-912

18. Szabo BK, Aspelin P, Wiberg MK, Boné B (2003) Dynamic MR imaging of the breast. Analysis of kinetic and morphologic diagnostic criteria. Acta Radiol 44(4):379-386

19. Kuhl CK (2007) Current status of breast MR imaging. Part 2. Clinical applications. Radiology 244:672-691

20. Belli P, Bufi E, Bonatesta A, Patrolecco F, Padovano F, Giuliani M et al (2016) Unenhanced breast magnetic resonance imaging: detection of breast cancer. Eur Rev Med Pharmacol Sci 20:4220-4229

21. Roknsharifi S, Fishman MDC, Agarwal MD, Brook A, Kharbanda V, Dialani V (2019) The role of diffusion weighted imaging as supplement to dynamic contrast enhanced breast MRI: can it help predict malignancy, histologic grade and recurrence? Acad Radiol 26:923-929

22. Santamaría G, Velasco M, Bargalló X, Caparrós $X$, Farrús B, Fernández LP (2010) Radiologic and pathologic findings in breast tumors with high signal intensity on T2-weighted MR images. Radiographics 30(2):533-548

23. Moschetta M, Telegrafo M, Rella L, Capolongo A, Stabile lanora AA, Angelelli G (2014) MR evaluation of breast lesions obtained by diffusion-weighted imaging with background body signal suppression (DWIBS) and correlations with histological findings. Magn Reson Imaging 32(6):605-609

24. Khaladkar SM, Kamal A, Kamal V, Kumar S, Singh G, Kalra R (2015) Pitfall in differentiation of hemorrhagic vs. fatty lesions in female pelvis using fat saturated sequences with inversion recovery - role of T1 FATSAT sequence - a case report with radiological review. IOSR J Dental Med Sci 14(3):86-90

25. Baltzer PAT, Benndorf M, Dietzel M, Gajda M, Camara O, Kaiser WA (2010) Sensitivity and specificity of unenhanced MR mammography (DWI combined with T2-weighted TSE imaging, ueMRM) for the differentiation of mass lesions. Eur Radiol 20:1101-1110

26. Telegrafo M, Rella L, lanora AAS, Angelelli G, Moschett M (2015) Unenhanced breast MRI (STIR, T2-weighted TSE, DWIBS): an accurate and alternative strategy for detecting and differentiating breast lesion. Magn Reson Imaging 3:951-955

27. Kul S, Oğuz S, Eyüboğlu I, Kömürcüoğlu Ö (2015) Can unenhanced breast MRI be used to decrease negative biopsy rates? Diagn Interv Radiol 21:287-292

28. Trimboli RM, Verardi N, Cartia F, Carbonaro LA, Sardanelli F (2014) Breast cancer detection using double reading of unenhanced MRI including T1weighted, T2-weighted STIR, and diffusion weighted imaging: a proof of concept study. AJR 203:674-681

29. Moschetta M, Telegrafo M, Introna T, Coi L, Rella L, Stabile lanora AA et al (2016) Role of STR and T2-weighted TSE MR sequences for recognizing occult inflammatory breast cancer. Eur Congress Radiol. https://doi.org/10.1594/ecr2016/C-2152

\section{Publisher's Note}

Springer Nature remains neutral with regard to jurisdictional claims in published maps and institutional affiliations. 